\title{
Mesalazine-induced Hypersensitivity Pneumonitis
}

\author{
Francisca M Pereira, Cristina Marques, Teresa Boncoraglio, Joana Esteves, Marinha Silva, Joana Braga, Márcia Ribeiro \\ Internal Medicine Department, Hospital Santa Maria Maior, Barcelos, Portugal
}

Doi: 10.12890/2021_002194 - European Journal of Case Reports in Internal Medicine - ๑ EFIM 2020

Received: 03/12/2020

Accepted: $11 / 12 / 2020$

Published: 07/01/2021

How to cite this article: Pereira FM, Marques C, Boncoraglio T, Esteves J, Silva M, Braga J, Ribeiro M. Mesalazine-induced hypersensivity pneumonitis EJCRIM 2021;8: doi:10.12890/2020_002194.

Conflicts of Interests: The Authors declare that there are no competing interests.

This article is licensed under a Commons Attribution Non-Commercial 4.0 License

\section{ABSTRACT}

A 57-year-old woman with Crohn's disease (ulcerative proctitis) treated with mesalazine (5-ASA) developed worsening respiratory distress and cough. The lack of response to antibiotics and the results of bronchoalveolar lavage led to the diagnosis of mesalazine-related hypersensitivity pneumonitis, an infrequent entity. Symptoms improved after discontinuation of mesalazine and the administration of corticosteroid therapy. The authors discuss the diagnosis and management of this rare condition.

\section{LEARNING POINTS}

- A diagnosis of mesalazine-related hypersensitivity pneumonitis should be considered when unexplained respiratory symptoms develop during treatment with mesalazine.

- It is important to distinguish pulmonary manifestations in patients with inflammatory bowel disease secondary to drug-related toxicity from the disease process itself.

- Amelioration of symptoms and improvement in imaging and lung function seem to occur only upon abrupt discontinuation of the drug; severe symptoms such as respiratory failure may justify corticosteroid therapy.

\section{KEYWORDS}

Mesalazine, ulcerative colitis, drug reaction, hypersensitivity pneumonitis, lung injury

\section{INTRODUCTION}

Mesalazine is a 5-ASA drug used to treat inflammatory bowel disease (IBD). Although it is usually well tolerated, associated toxicity has been described ${ }^{[1]}$ and eosinophilic, organizational or non-specific interstitial pneumonia can affect the lungs. Mesalazine-induced hypersensitivity pneumonitis is rare ${ }^{[2]}$ but should be considered when unexplained respiratory symptoms and fever develop in patients treated with mesalazine. Withdrawal of the drug usually results in remission of the pulmonary side effects ${ }^{[3]}$.

\section{CASE DESCRIPTION}

Our patient was a 57-year-old woman with a history of type 2 diabetes mellitus, inflammatory bowel disease (ulcerative proctitis) and idiopathic thrombocytopenic purpura (IPT) for which she had undergone splenectomy. She was medicated with gliclazide 60 mg/ day, mesalazine $3 \mathrm{~g} /$ day and eltrombopag $25 \mathrm{mg}$ every other day. She presented to the emergency department with an 8-day history of progressively worsening dyspnoea on medium exertion, associated with an irritable cough and asthenia. She denied fever, chest pain, nocturnal hyperhidrosis, anorexia, weight loss or changes in intestinal transit.

On admission, the patient was haemodynamically stable and apyretic with a peripheral oxygen saturation of $95 \%$ in room air. Pulmonary auscultation revealed bilateral and symmetrical breath sounds, wheezing and rare but diffuse crackles. 
An electrocardiogram was performed and showed a sinus rhythm with no changes suggestive of ischaemia. Blood analysis demonstrated elevated inflammatory parameters (leucocytes 16,200/l, neutrophils $76.9 \%$ and C-reactive protein 7.73 mg/dl), positive D-dimers and normal renal function, with no other major changes. Arterial blood gas analysis revealed normal gas exchange without respiratory failure. As the chest x-ray (Fig. 1) showed bilateral heterogeneous diffuse infiltrate, a chest CT scan was requested and revealed extensive bilateral ground-glass opacification (Figs. 2 and 3). The findings were unspecific and atypical pneumonia was a diagnostic hypothesis. Pulmonary thromboembolism was excluded. The patient was discharged with a course of antibiotics (amoxicillin/clavulanic acid plus azithromycin).
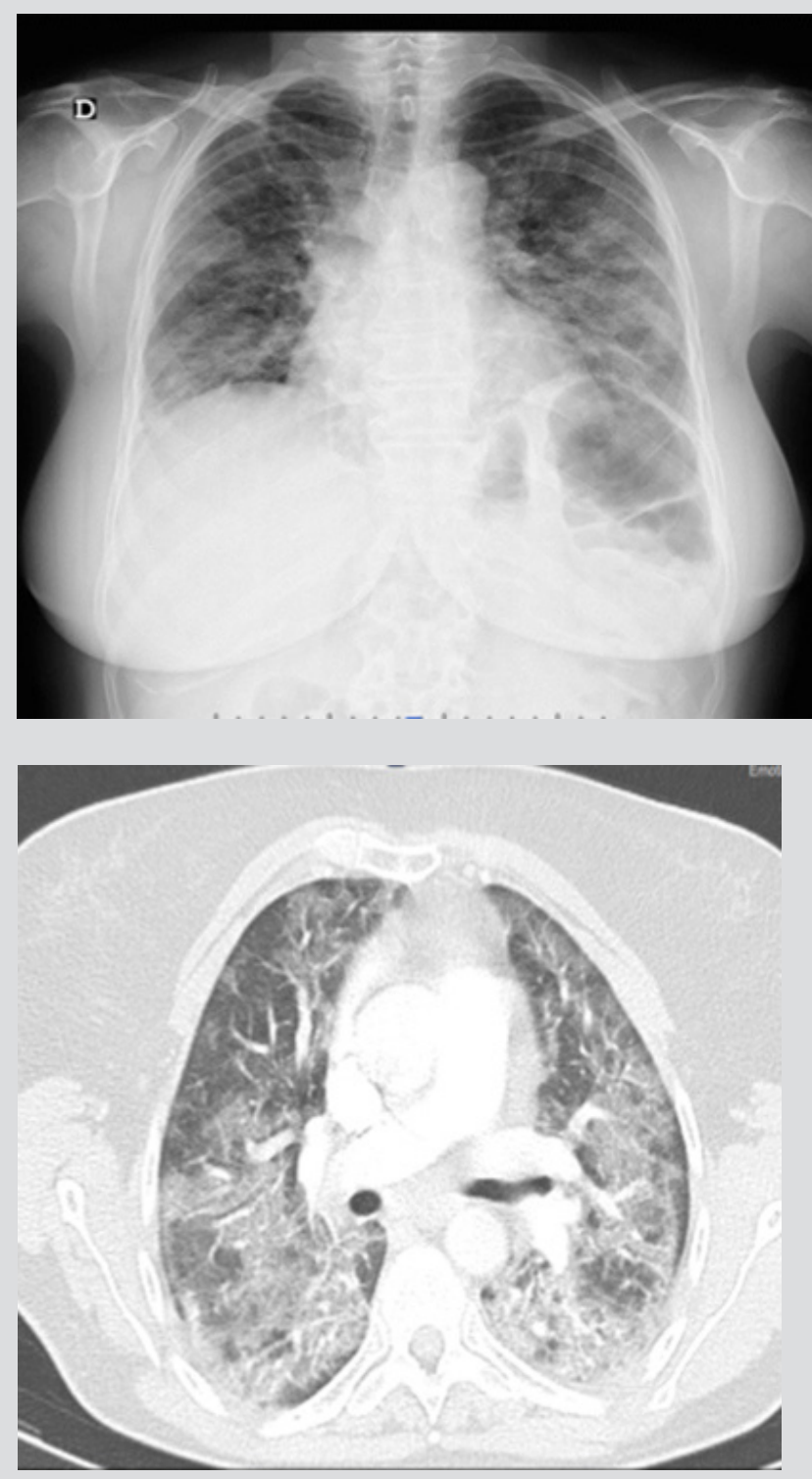

Figure 2. Chest CT scan (axial view) showing bilateral ground-glass opacities, with subsegmental consolidation in basal topography
Figure 1. Chest $x$-ray revealing interstitial heterogeneous diffuse infiltrate
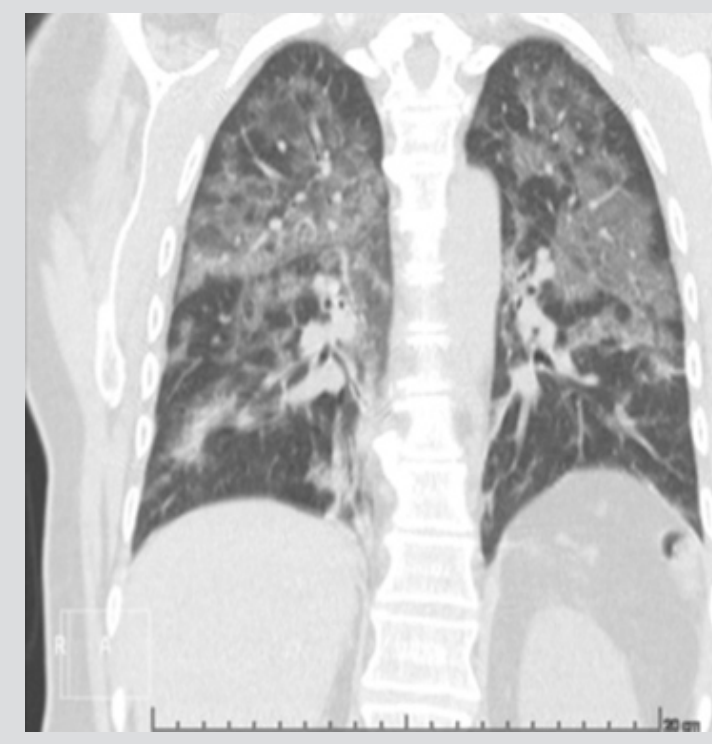

Figure 3. Chest CT scan (coronal view) showing bilateral ground-glass opacities, with sub-segmental consolidation in basal topography

One week after discharge, the patient returned to the emergency department due to worsening dyspnoea on mild exertion with no other associated complaints. Upon admission, she presented polypnoea on slight muscle movement. The rest of the physical examination was unchanged. Blood gas analysis showed respiratory alkalaemia and hypoxaemic respiratory distress, with a $\mathrm{PaO}_{2} / \mathrm{FiO}{ }_{2}$ ratio of 200 . The patient was admitted and antibiotic therapy was changed to a 10-day course of piperacillin/tazobactam. However, respiratory failure and fever did not resolve. Blood cultures were negative as were antigen tests for pneumococcus and legionella. Sputum analysis was not performed. Serology for atypical microorganisms was negative. 
Videobronchofibroscopy with bronchoalveolar lavage (BAL) revealed intense neutrophilic and eosinophilic lymphocytic alveolitis. Flow cytometry of lymphocyte subpopulations revealed a predominance of CD8+ T cells. Testing for acid-resistant bacilli was negative. Culture and cytology for malignant cells was negative. Due to suspicion of mesalazine hypersensitivity pneumonia, it was decided to discontinue mesalazine and a course of corticosteroid therapy was initiated with improvement of symptoms, resolution of respiratory failure and regression of the inflammatory parameters.

\section{DISCUSSION}

A thorough anamnesis, lack of response to antibiotics, and BAL results led to the likely diagnosis of mesalazine hypersensitivity pneumonia in this patient. The most common clinical-pathological entity in such cases is interstitial pneumonitis with a predominance of lymphocytes or eosinophils and a reduced BAL CD4+/CD8+ ratio ${ }^{[1]}$. However, the pathogenesis of lung injury induced by mesalazine remains uncertain. Proposed causes include immune-mediated alveolitis caused by lymphocyte stimulation, dose-dependent lung injury, direct toxic insult and oxidative damage to the pulmonary epithelium ${ }^{[3]}$. It is important to distinguish between pulmonary manifestations of IBD and those secondary to drug toxicity. Pulmonary involvement in IBD is a common complication, usually presenting with impaired lung function ${ }^{[4]}$.

Lung biopsy is often non-specific and findings mimic those of other conditions, requiring clinical, laboratory and radiological confirmation. However, it can be a useful tool to exclude interstitial disease and infection, and to document the pattern of lung injury and the underlying disease. However, it has rarely been performed in cases described in the literature ${ }^{[3]}$. Resolution of symptoms and improved radiological findings have been documented days to weeks after drug withdrawal, often with total recovery of pulmonary function ${ }^{\text {[5] }}$

Our case shows that although uncommon, this entity should be considered in patients receiving mesalazine who develop unexplained respiratory symptoms without another cause documented. Severe symptoms such as respiratory failure can justify corticosteroid therapy ${ }^{[2]}$, as in the present case. The diagnostic challenge of the case, as well as the rarity of descriptions in the literature of pulmonary toxicity caused by mesalazine, prompted the authors to present this report.

\section{REFERENCES}

1. Huang PH, Kuo CJ, Lin CW, Cheng YM, Hu HC, Lin CY, et al. Mesalazine-related lung disease in a patient with ulcerative colitis: a case report. Medicine (Baltimore) 2018;97(48):36.

2. Kacprzak A, Siemion-Szcześniak I, Szturmowicz M, Bestry I, Langfort R, Kuś J. Pulmonary pathology in patients with ulcerative colitis treated with mesalazine-a challenging and complex diagnostic problem. Case series and literature review. Pneumonol Alergol Pol 2014;82(4):368-376.

3. Kotsiou OS, Gourgoulianis KI. A case report of mesalazine-induced lung injury: a reversible drug side effect. Respir Med Case Rep 2019;27:100865.

4. Fouka E, Stefanopoulo P, Dramba V, Bombotas D. Acute interstitial lung disease in a patient with ulcerative colitis: case report and literature review. Pneumon 2012;25(3):320324.

5. Bitton A, Peppercorn MA, Hanrahan JP, Upton MP. Mesalamine-induced lung toxicity. Am J Gastroenterol 1996;91(5):1039-1040. 